\title{
TRADUCCIÓN
}

\section{LA ADQUISICIÓN DE LOS LIBROS ELECTRÓNICOS EN LAS BIBLIOTECAS DE LOS CENTROS DE ENSEÑANZA SUPERIOR DE SUECIA*}

\author{
Elena Maceviciute, Martin Borg, Ramune Kuzminiene y Katie Konrad \\ Universidad de Borås. Suecia.
}

\begin{abstract}
Resumen: El objetivo de este artículo es comparar las ventajas y problemas de la adquisición de libros electrónicos identificados en la literatura de investigación a los experimentados por dos bibliotecas universitarias suecas. Como método de trabajo se utilizó una revisión de la literatura para identificar los principales problemas relacionados con la adquisición de los libros electrónicos en las bibliotecas universitarias. Los datos para la comparación se recogieron a través de estudios de caso en dos universidades suecas. Se utilizaron y analizaron documentos, entrevistas y experiencias personales en la recolección de datos. Entre los resultados destaca que los principales impulsores de la adquisición de libros electrónicos de las bibliotecas universitarias suecas son las necesidades percibidas en los usuarios. Los libros electrónicos son considerados como potencialmente útiles para resolver algunos de los problemas de servicio de la biblioteca. Un considerable número de desafíos y problemas identificados en los estudios de casos coinciden con los que se derivan de la revisión de la literatura. Los problemas de la adquisición de libros electrónicos en las bibliotecas universitarias parecen ser comunes entre los países occidentales económicamente fuertes. Los bibliotecarios universitarios ven ciertas ventajas en los libros electrónicos para sus usuarios y las bibliotecas. Los editores y bibliotecarios académicos esperan que los libros electrónicos no pierdan las ventajas que ofrecen los libros impresos. Los editores limitan el uso de los libros electrónicos para asegurarse los ingresos como en el caso de la venta de copias individuales impresas. Los bibliotecarios tratan de recuperar el mismo nivel de control sobre las colecciones de libros electrónicos del que disponían en los materiales impresos.
\end{abstract}

Palabras clave: activos de información digital; bibliotecas universitarias; desarrollo de colecciones bibliotecarias; libros electrónicos; mercado de la información; Suecia.

\begin{abstract}
Title: THE ACQUISITION OF E-BOOKS IN THE LIBRARIES OF THE SWEDISH HIGHER EDUCATION INSTITUTIONS.

Abstract: Our aim is to compare the advantages and problems of e-book acquisition identified in research literature to those experienced by two Swedish university libraries. A literature review was used to identify the main issues related to acquisition of e-books by academic libraries. The data for comparison were collected through case studies in two Swedish universities. Document analysis, interviews and personal experience were used for data collection. The main drivers of e-book acquisition by Swedish academic libraries are the perceived needs of the users. E-books are regarded as potentially useful for solving some of the problems of library service. A number of challenges and problems identified by the participants in the case studies coincide with those that were derived from the literature review. The problems of e-book acquisition in academic libraries seem to be common to the economically strong Western countries. University librarians see certain advantages of e-books for their users and libraries. Publishers and academic librarians expect that e-books would not lose the advantages that printed books offered to them. Hence, publishers restrict the usage of e-books to ensure revenues as if from selling individual copies. Librarians try to regain the same level of control over e-book collections as for printed materials.

Keywords: digital information assets; academic libraries; libraries collections development; e-books; information market; Sweden.
\end{abstract}

\section{INTRODUCCIÓN}

El número de artículos y libros en formato de libro electrónico está creciendo rápidamente, pero esta literatura permanece fragmentada y desperdigada entre varias disciplinas. Una de las áreas que ya pueden beneficiarse de la consolidación de datos de investigación es la de las bibliotecas universitarias. Estas bibliotecas adquieren libros electrónicos y manejan una amplia variedad de colecciones de un número de distribuidores, agregadores y editores, usando la rica experiencia adquirida en las negociaciones para la adquisición de las revistas electrónicas y bases de datos de artículos de texto completo, así como otros recursos digitales. El suministro de estos recursos en formato de libro electrónico (principalmente manuales de clase, monografías científicas, tratados, informes de investigación,

\footnotetext{
* Traducción del artículo original publicado en la revista Information Research: an international electronic journal. Traducción y publicación autorizada por los autores y el editor de la revista.

Traducido por Francisco Javier Martínez Méndez, profesor de Biblioteconomía y Documentación de la Universidad de Murcia.

Maceviciute, E.; Borg, M.; Kuzminiene R. y Konrad, K. The acquisition of e-books in the libraries of the Swedish higher education institutions. Information Research, 2014, vol. 19, $\mathrm{n}^{\circ}$ 2. Disponible en: <http://InformationR.net/ir/19-2/paper620.html> Consulta: [26 de marzo de 2015].

MACEVICIUTE, E.; BORG, M.; KUZMINIENE, R. y KONRAD, K. La adquisición de los libros electrónicos en las bibliotecas de los centros de enseñanza superior de Suecia. Anales de Documentación, 2015, vol. 18, $\mathrm{n}^{\circ} 1$. Disponible en: http://dx.doi.org/10.6018/analesdoc.18.1.224341
} 
libros de referencia y algunos otros libros no de ficción que son utilizados como bibliografía de apoyo al estudio), en idioma inglés ya es bastante prolífico en el mercado internacional y tiene gran importancia para la comunicación académica. Incluso la producción de monografías electrónicas en los idiomas locales (de alcance más reducido), surge en muchos países (por ejemplo: Suecia, Polonia, etc.) (Bernhardsson, et al., 2013) y (Kulczycki, 2012) y en diferentes contextos editoriales (editoriales universitarias, fundaciones sin ánimo de lucro, editoriales comerciales). Estos textos también llaman la atención sobre el papel de las bibliotecas universitarias en su producción, distribución y conservación. Como las bibliotecas universitarias fueron las principales entidades compradoras de monografías académicas que rara vez aparecían en el mercado como productos enteramente comerciales, la presión que se ejerce sobre el presupuesto de las bibliotecas para la adquisición de otros recursos electrónicos (revistas, bases de datos, libros de texto, etc.), altera de forma severa el modelo de negocio existente en la producción de monografías (Svensson y Eriksson, 2013), por lo que se buscan nuevas formas para su distribución. ${ }^{1}$ El objetivo de este trabajo es comparar los problemas identificados en la literatura científica con los experimentados por dos bibliotecas universitarias suecas, lo que debería ayudar a establecer, en primer lugar, si los problemas generales identificados en la literatura científica de otros países están presentes en una biblioteca universitaria sueca y, en segundo lugar, determinar cuáles son las necesidades expresadas por estas bibliotecas para adquirir y proporcionar acceso a los libros electrónicos y, finalmente, ¿cuáles son los problemas que experimentan en relación con este tema?

Los resultados de la revisión de la literatura científica y del análisis de dos estudios de caso se utilizarán en el diseño de un estudio más amplio a desarrollar en las bibliotecas universitarias de Suecia sobre la práctica habitual de adquisición y gestión de libros electrónicos en el seno de un proyecto de investigación financiado por el Consejo Sueco de Investigación. Los autores trabajan o estudian en una de las universidades objeto de estudio (en la Universidad de Borås), y en el caso de la autora principal, es además la responsable de un proyecto de investigación financiado. El tema de estudio se limita a las cuestiones de la adquisición de los libros electrónicos y a la gestión de las colecciones en este formato, no incluye el uso de libros electrónicos o las perspectivas de los usuarios de libros electrónicos en las bibliotecas o investigaciones de los proveedores de libros electrónicos para bibliotecas universitarias académicas.

\section{METODOLOGÍA}

El Modelo de Winston (1998) de innovación tecnológica en los medios ha ofrecido un marco útil para la presente investigación. En otro trabajo (Bergström, Höglund, Maceviciute, Nilson y Wilson, 2014) explican el uso de este modelo para explorar la difusión de libros electrónicos de la siguiente manera:

"Winston sugiere que, antes de que se pueda emplear una invención, debe existir un determinado número de prototipos en diversos formatos (tales como "rechazados", "aceptados", "paralelos" y "parciales") que surgen en el campo tecnológico a partir de ideas derivadas de la ciencia. Para que la invención sea adoptada como necesidad social sobrevenida y se considere precisa, pueden hacer falta otros factores tecnológicos que ayuden en esa difusión, o la concentración de fuerzas sociales que la apoyen o, directamente, que exista un interés comercial. Winston cita el caso de la única vía férrea que necesitaba el ferrocarril y que se perdía más allá de la línea del horizonte, su desarrollo alentó la adopción del telégrafo como medio de comunicación omnipresente en la época. La amplia difusión de una innovación requiere que los intentos de suprimir el radical potencial ${ }^{2}$ de la misma deben ser superados: podemos tomar como ejemplo los intentos de la industria editorial de la música para suprimir por diversos medios, incluida la gestión de derechos digitales, la invención de los archivos digitales de música. Por último, algunas invenciones pueden demostrar que son redundantes con el tiempo y otras invenciones subsidiarias pueden escindirse de la invención objeto de estudio". (Bergström, et al., 2014).

El modelo incluye cuatro etapas de la difusión de la innovación: ideación, prototipado, la invención y la difusión propiamente dicha (la producción). La etapa de la invención transforma algunos de los prototipos existentes en invenciones bajo las premisas de ciertas necesidades sociales sobrevenidas (es decir, un complejo de fuerzas tecnológicas y sociales que trabajan directamente en el proceso de innovación). La etapa final se refiere a una innovación en movimiento dentro del mercado, de acuerdo con las circunstancias de la supresión de su potencial radical (Winston, 1998, p. 7-10). El modelo ofrece la posibilidad de explorar la tensión existente entre el potencial radical del libro electrónico en conflicto con la inversión establecida en las tecnologías existentes y la adhesión a los modelos de negocio establecidos. Las necesidades sociales subyacentes han afectado al desarrollo de tecnologías que sustentan los dispositivos de lectura electrónica y de difusión de los libros electrónicos en una variedad de formatos y en diferentes esferas. Las necesidades sociales subyacentes de los libros electrónicos podrían ser rastreadas a través de las formas y condiciones de uso de los libros electrónicos en estos distintos ámbitos. La exploración de las actividades de los diferentes actores relacionados con la explotación de los libros electrónicos también puede revelar los medios de la supresión del potencial radical asociado. En la actualidad, observando la difusión del libro electrónico a nivel 
mundial y la atención que actualmente recibe del sector editorial del libro, podemos considerar que ha superado las etapas de prototipado y de invención, habiendo entrado de pleno en la etapa de producción.

Dos han sido los métodos de trabajo seguidos en el desarrollo de esta investigación: la revisión de la literatura científica y el desarrollo de estudios de caso. En un primer momento se realizó una búsqueda bibliográfica en las bases de datos ISI Web of Knowledge y Scopus, búsqueda ampliada con los hallazgos en otras fuentes (por ejemplo, utilizando los catálogos de bibliotecas suecas y el buscador académico scholar.google.se). La revisión de la literatura científica relevante se centra tanto global como localmente (a nivel de Suecia), sobre el impacto del libro electrónico en las bibliotecas universitarias. Como es lógico, hay más información disponible a escala global, por lo tanto, la mayor parte de los ejemplos e investigaciones provienen de esta literatura, generalmente producida en idioma inglés. No es nuestra intención restar importancia al caso particular sueco, pero la falta de material publicado impide un análisis igualmente profundo. Las publicaciones utilizadas en esta revisión ya fueron analizadas en dos tesis de máster sobre la presencia de los libros electrónicos en las bibliotecas universitarias (Konrad, 2013) y (Kuzminiene, 2014). El objetivo principal de esta revisión era comprender las tendencias contemporáneas en la adquisición de los libros electrónicos y el desarrollo de colecciones en este formato, desde la perspectiva de la biblioteca universitaria. Nuestro estudio se ha centrado en las publicaciones aparecidas en los últimos siete u ocho años, que han significado un cambio en el uso y difusión de los libros electrónicos, aunque algunos trabajos más antiguos también han sido utilizados. La revisión de la literatura científica refleja los temas identificados a partir de las cuestiones más amplias y poco a poco permite introducir temas más concretos.

En el primer caso de estudio se obtuvo información de la biblioteca, a partir de eventos celebrados en la misma y de entrevistas con el personal bibliotecario encargado de la adquisición de estas publicaciones. También se incluye en esta primera parte del estudio la experiencia directa de uno de los autores de este artículo que trabaja en la biblioteca precisamente en la gestión de la colección de libros electrónicos y las negociaciones de licencias. Los datos fueron recogidos durante los últimos meses del año 2013 y el inicio del año 2014. El segundo caso de estudio fue realizado por Katie Konrad entre los años 2012 y 2013 analizando la literatura de investigación, anotando datos puntuales y observando los resultados presentados en esos trabajos previos.

\section{EL LIBRO ELECTRÓNICO COMO CONCEPTO Y COMO UN OBJETO DIGITAL}

Los estudiosos han utilizado muchas maneras diferentes de definir el libro electrónico. Armstrong y Lonsdale (2005) afirman que en un principio hubo cierta confusión resultante por la asociación de la publicación en sí con el lector de libros (el dispositivo de lectura). En la actualidad, la mayoría de las definiciones hacen hincapié en una estructura similar a un libro y a la posibilidad de la lectura de un texto en la pantalla de un dispositivo digital (Armstrong, 2014).

Hace ya algún tiempo que un libro electrónico es algo más que una versión en PDF del libro impreso, ya que "como textos digitales pueden cambiar y aumentar la naturaleza de los objetos de libros como en un contexto electrónico" (Nelson, 2008). Vassileiou y Rowley sugieren una definición en dos partes: la primera se refiere a la naturaleza razonablemente estable de los libros electrónicos (características persistentes) y la segunda se centra en los cambios de la tecnología:

"(1) Un libro electrónico es un objeto digital con textos y/o otros contenidos, que surge como resultado de la integración del concepto familiar de un libro con las características que se pueden proporcionar en un entorno electrónico. (2) Los libros electrónicos, por lo general, tienen características en uso, como las funciones de búsqueda y de referencia cruzada, hipervinculos, marcadores, anotaciones, resaltados, objetos multimedia y herramientas interactivas". (Vassiliou y Rowley, 2008, p. 362).

Al igual que ocurre con los libros impresos, también hay muchos géneros literarios editados en formato de libro electrónico, variedad que se multiplica más que la tecnología que los implementa. Los libros electrónicos editados varían desde copias escaneadas de libros impresos a estructuras de tipo de base de datos (Minčić-Obradovic, 2011). Según Tedd (2005) en las bibliotecas universitarias las colecciones de libros electrónicos (generalmente adquiridos), incluyen libros de texto, libros multimedia, libros de referencia y directorios, así como versiones digitalizadas de libros fuera de catálogo. Del mismo modo, Armstrong y Lonsdale (2005) en su estudio de las bibliotecas de educación del Reino Unido, proporcionan una lista casi idéntica para el tipo de libro electrónico predominante en esas bibliotecas universitarias, añadiendo las monografías académicas, textos de literatura gris y libros electrónicos gratuitos. Como se ve fácilmente, estas listas no son congruentes e incluyen elementos definidos por género (monografías académicas, libros de texto, libros de referencia), por el modo de acceso (libros electrónicos gratuitos, literatura gris, libros fuera de catálogo), o por las características tecnológicas implementadas (multimedia, bases de datos). El asunto de los "libros electrónicos" académicos puede abordarse en relación con el tema del libro electrónico como un concepto. Sin 
embargo, aquí se abordará de manera pragmática, considerando un libro electrónico académico como cualquier forma digital de un libro (cumpliendo así los términos de la definición de Vassileiou y Rowley), adquiridos por las bibliotecas universitarias.

\subsection{Formatos de libros electrónicos y plataformas}

Contrariamente a la situación de los artículos publicados en las revistas electrónicas, donde predomina el formato PDF, no existe un formato de archivo estándar para los libros electrónicos. Según Walters (2013a), algunas fuentes afirman que existen entre 16 y 27 formatos de libros electrónicos diferentes actualmente en uso. Lo cierto es que el formato de archivo PDF parece predominar las colecciones de las bibliotecas universitarias (Vasileiou, Hartley y Rowley, 2012a) y (Walters, 2013a). La mayoría de los formatos de datos propietarios almacenan en las formas y maneras diseñadas por una empresa particular, por lo que esos datos son solo fácilmente accesibles cuando se utiliza el software y el hardware de la compañía propietaria. La especificación de formato de codificación de los datos no se libera habitualmente, o bien se publica de forma restringida por la gestión de derechos digitales (DRM). Esto significa que al adquirir un libro electrónico en formato propietario, se bloquea al cliente en una determinada plataforma o con un lector específico de libros electrónicos y viceversa. Es natural que las bibliotecas universitarias sean reacias a invertir en tecnologías múltiples para cada libro electrónico aunque si el contenido está disponible sólo en una plataforma específica, las bibliotecas universitarias se podrían ver obligadas a comprar de todos modos "y pronto se encontrarían en la situación de tener más plataformas de lo que hubieran deseado" (Thompson y Sharp, 2009, p. 138). Hay que tener en cuenta que los formatos PDF son también a menudo protegidos por la gestión de derechos digitales.

No hay duda de que las diferencias en los formatos causan una serie de diversos problemas, no solo para los usuarios de los libros electrónicos, sino también para los bibliotecarios. Nelson (2008) habla de la reducción de las tasas de adopción por parte de estudiantes y personal académico, así como de las barreras que la variedad de formatos de archivos de libros electrónicos crean. Del mismo modo, Walters afirma que "la multiplicidad de formatos de archivos de libros electrónicos plantea serias dificultades tanto para la compatibilidad entre plataformas como el acceso a largo plazo" (2013b, p. 203). El surgimiento de nuevos formatos y plataformas múltiples de editores también está afectando el flujo de trabajo de las bibliotecas, ya que tienen que ajustar o establecer nuevos procedimientos para el manejo de los libros electrónicos y el personal debe manejar la creciente complejidad del sector (Thompson y Sharp, 2009) y (Morris y Sibert, 2010). Los formatos antiguos pueden llegar a ser incompatibles con los lectores actualmente existentes, o incluso peor, podrían llegar a ser abandonados por los proveedores de libros electrónicos, dejándolos fuera del mercado y causando grandes problemas para el acceso y la preservación de los recursos ya adquiridos en estos formatos (Walters, 2013a).

\section{DESARROLLO DE COLECCIONES Y LIBROS ELECTRÓNICOS EN LAS BIBLIOTECAS UNIVERSITARIAS}

Según Tedd (2005), el desarrollo de las versiones electrónicas de los libros impresos puede ser visto como parte de todo el fenómeno de la publicación digital, que comenzó en la década de los sesenta. Sin embargo, la mayoría de los investigadores datan la primera aparición de los libros electrónicos en las bibliotecas universitarias en la década de los noventa (Armstrong y Lonsdale, 2005), (Minčić-Obradović, 2011) y (Chell, 2010). Los años 2007-2009 marcan una transición para el mercado de los libros electrónicos de educación superior con un mayor crecimiento tanto en la publicación de libros electrónicos como en el desarrollo de colecciones y bibliotecas digitales (Nelson, 2008). Los libros electrónicos han entrado a formar parte de la corriente principal de adquisición de libros para las principales bibliotecas universitarias, sobre todo, cuando la variedad y el número de dispositivos de lectura de los libros electrónicos aumentó la demanda de libros en este formato por parte de los usuarios, y también por una mayor provisión de libros electrónicos por parte de los editores (Beisler y Kurt, 2012).

Hay muchos aspectos de los libros electrónicos (y sobre sus colecciones en el seno de bibliotecas universitarias), que han sido investigados en los últimos años. Blummer y Kenton (2012) proporcionan una revisión de la literatura sobre las mejores prácticas de gestión de libros electrónicos en las bibliotecas universitarias que cubre desde el año 2005. Otros investigadores estaban interesados en el uso de las colecciones de libros electrónicos; diferentes estudios analizan el uso académico de los libros electrónicos por parte de diferentes usuarios (Martin y Quan-Haase, 2013), (Ahmad y Brogan, 2012) y (Cassidy, Martínez y Shen, 2012); y hay estudios que buscan dentro de las percepciones de los usuarios de libros electrónicos, así como el modo de utilización de los mismos. Esta revisión muestra que las experiencias de las bibliotecas universitarias en la adquisición de los libros electrónicos, y en su gestión, han atraído la atención de los investigadores. Incluso algunos estudios de usuarios y uso avisan sobre la multitud de cuestiones que afectan a la adquisición de libros electrónicos (por ejemplo el trabajo de Sheperd y Arteaga del año 2014). Sin embargo, todavía hay muy poco análisis e investigación en profundidad sobre la gestión de las colecciones y los 
problemas de adquisición de libros electrónicos en las bibliotecas universitarias. Vasileiou, Rowley y Hartley (2012c) creen que los bibliotecarios se enfrentan a un conjunto de retos y problemas en la gestión de libros electrónicos, el desarrollo de colecciones y la gestión del ciclo de vida del libro electrónico (desde la disposición del presupuesto para la compra hasta la retirada, si se llevara a cabo, del libro).

La incorporación de un nuevo medio en la biblioteca puede ser una tarea difícil, que requiere el desarrollo e implementación de políticas específicas y planes de acción bien establecidos (Stamison, 2011). Blummer y Kenton (2012) están de acuerdo en que son los planes y las estrategias de adquisición las que permiten garantías de éxito, así como la integración de los libros electrónicos en las colecciones de la biblioteca. Idealmente, los problemas de gestión de cobro alrededor de la selección, adquisición y promoción de los libros electrónicos tienen que abordarse dentro de las políticas de desarrollo de colecciones de una institución. Sin embargo, un nuevo medio crea siempre diferentes incertidumbres que impiden a las bibliotecas documentar debidamente las nuevas normas, en especial, en aquellas áreas donde los cambios pueden ser bastante rápidos. El reciente estudio de investigación sobre las experiencias y percepciones de la gestión del libro electrónico en las bibliotecas universitarias del Reino Unido muestra que existen políticas relacionadas con el libro electrónico y que incluyen en las políticas de desarrollo de colecciones de las bibliotecas en general. Sin embargo, no existen aún políticas de gestión específicas de la colección de libros electrónicos, ni siquiera está previsto en las bibliotecas encuestadas (Vasileiou et al., 2012c).

\subsection{Barreras para el acceso y uso de los libros electrónicos en las bibliotecas universitarias}

Al observar el actual mercado del libro, parece que no hay escasez de libros electrónicos en cualquier área. La mayor parte de los editores científicos incluyen libros electrónicos en sus catálogos y en sus sitios web, si bien tratar de encontrar los títulos más recientes en este formato en la mayoría de ellos es bastante complicado. En realidad, la baja disponibilidad de los libros electrónicos académicos (o la no disponibilidad) es uno de los principales problemas para los bibliotecarios encargados de construir estas colecciones digitales. Los libros electrónicos se han convertido en algo habitual para los editores y vendedores de títulos populares, y aunque la cantidad de textos académicos y científicos está creciendo, el mercado académico los sigue recibiendo de forma bastante pobre (Walters, 2013b). Dewan (2012) señala que este problema se convierte en uno de los mayores obstáculos para el desarrollo de las colecciones de libros electrónicos en las bibliotecas universitarias y de investigación. Sin embargo, este lento ritmo de crecimiento de la cantidad de libros electrónicos académicos es sólo una parte del problema.

\subsubsection{Embargos}

En muchos casos hay retrasos en el lanzamiento de los libros electrónicos académicos con el fin de proteger las ventas de las versiones impresas (generalmente en tapa dura) ya que aportan mayores ingresos tras su etapa de producción (Meehan, 2010). Esto se observa en el mercado de los principales libros de texto donde los editores no se apresuran a invertir en el desarrollo de materiales en formato de libro electrónico, tal como señalaban Bennett y Landoni (2005). Hodges, Preston y Hamilton explican bien cómo funciona este modelo de negocio:

"cuanto mayor sea la edición de tapa dura es la única fuente de contenido, más dinero gana el editor; después del pico de ventas de la versión impresa en tapa dura, suele publicarse una edición de bolsillo y, en algún momento, la editorial publica el libro electrónico, primero a la venta, y más tarde concede el permiso para incluir el libro electrónico en colecciones alquiladas". (Hodges et al., 2010, p. 198).

Según algunos autores, este modelo de negocio obvia una gran ventaja del libro electrónico: la reducción del tiempo que ha de transcurrir entre la aceptación del manuscrito y la distribución pública formal de los ejemplares impresos (Walters, 2012), se pierde ahí esta ventaja. El período de embargo puede oscilar entre tres y dieciocho meses (Hodges et al., 2010) y (Walters, 2013b). Esto convierte en un gran dilema para los bibliotecarios su adquisición, ya que no muchas bibliotecas universitarias pueden permitirse el lujo de comprar el mismo contenido dos veces. Por lo tanto, tienen que tomar una decisión: retrasar la compra del título hasta que se libere el libro electrónico o bien comprar un libro de papel en su lugar. En algunos casos, las bibliotecas pueden permitirse el lujo de esperar a que el libro esté en formato electrónico, pero cuando un texto es considerado como material de lectura altamente necesario para una asignatura "tal demora es inaceptable" (Medeiros, 2011, p. 161). La opción de adquirir ediciones de bolsillo podría poner en peligro la configuración de la colección de libros electrónicos, especialmente en aquellas bibliotecas que favorecen el préstamo de las versiones en este formato. En estas bibliotecas esto también podría significar dejar a sus usuarios sin su formato preferido de lectura.

\subsubsection{Gestión de derechos digitales (DRM)}

La gestión de derechos digitales consiste realmente en la implantación de una "tecnología de control de acceso utilizada por los titulares de derechos de autor para limitar el uso de contenidos digitales" (Stamison 2011, p. 10). Esta gestión puede permitir o denegar el derecho de transferir el contenido de un dispositivo a otro, y también establece los valores máximos para imprimir, copiar, compartir y descargar para leer sin conexión. A veces, las 
restricciones de gestión de derechos digitales son en realidad contrarias a los términos de la licencia y "en la práctica esto significa, que el DRM da a los editores la capacidad inicial para limitar el uso sin embargo”. (Walters, $2013 \mathrm{~b}, \mathrm{p}$. 5). No es sorprendente que estos límites frustren y hasta enfurezcan a los usuarios de la biblioteca. Joe Wikert, editor y autor del blog Publishing 20203, también comparte una visión muy crítica sobre la gestión de derechos digitales. Argumenta que no elimina la piratería, y que la presencia de muchas restricciones no sólo hace que el libro electrónico sea menos atractivo que los libros de papel, sino que también implica una falta de confianza (Wikert, 2012). Varios sistemas de gestión de derechos digitales se utilizan para proteger los derechos de autor del propietario. En el ejercicio de esta protección, la gestión de derechos digitales limita el potencial de un libro electrónico reduciendo sus posibilidades de uso justo, pudiendo copiar las partes del texto para uso individual y educativo sin infringir los derechos económicos de los propietarios del copyright. La creciente impopularidad de la gestión de derechos digitales ha dado lugar al desarrollo y uso de medios alternativos, menos notorios, como las marcas de agua o sistemas de seguimiento de copias personalizadas de libros electrónicos (Srivastava y Sharma, 2012).

\subsubsection{Limitaciones relativas al préstamo interbibliotecario}

El préstamo interbibliotecario, tanto dentro de un país como a nivel internacional, es una de las formas de mejorar el uso de los recursos desarrollados por las bibliotecas, al mismo tiempo que participa en la gestión de sus colecciones a través del tiempo. Los libros electrónicos poseen un gran potencial para facilitar el proceso de préstamo interbibliotecario. Mediante el uso de un libro electrónico para esta función tradicional, las bibliotecas podrían ahorrar tiempo y dinero y también evitar situaciones en las que los usuarios de la biblioteca se puedan quedar sin un libro por el período de tiempo en que esté en préstamo a otra biblioteca. A pesar de estos beneficios, en la actualidad hay muchas barreras para el intercambio de los libros electrónicos, especialmente aquellos que están bajo la protección de los derechos de autor. Algunas licencias de libros electrónicos imponen una prohibición total del préstamo, mientras que otras permiten el intercambio de capítulos de libros con ciertos límites y restricciones (Radnor y Shrauger, 2012) y (Walters, 2013b). Otras restricciones adicionales incluyen el uso de una plataforma específica o de un formato propietario que limita los libros electrónicos que pueden ser prestados porque deben leerse en dispositivos específicos: "al igual que en muchos otros casos, las limitaciones impuestas por los proveedores niegan las ventajas que los libros electrónicos de otro modo podrían proporcionar". (Walters, 2013b, p. 6).

Algunas investigaciones muestran que las complejidades relacionadas con la comunicación y las limitaciones que participan en el intercambio de los libros electrónicos son tan molestas que los bibliotecarios, a pesar de que perciben el potencial existente y desean explotarlo, se abstengan de utilizar los libros electrónicos en las transacciones de préstamo interbibliotecario (Fredriksen, Cummings, Cummings y Carroll, 2011). Radnor y Shrauger comparten la misma opinión al ilustrar una situación común en las bibliotecas que tienen que hacer frente a las peticiones de libros electrónicos teniendo en cuenta las cuestiones de licencias, plataformas, flujos de trabajo adicionales y tiempo:

"estas barreras, para el mal de los libros electrónicos, van mucho más allá del ámbito del préstamo y provocan responder a muchos practicantes de este servicio que simplemente los libros electrónicos no están disponibles a través de préstamo interbibliotecario, o incluso desviar todas las peticiones de forma automática”. (Radnor y Shrauger, 2012, p. 156).

\section{ADQUISICIÓN DE LIBROS ELECTRÓNICOS EN LAS BIBLIOTECAS UNIVERSITARIAS}

Walters (2013b) afirma que los desafíos asociados con los libros electrónicos académicos son mucho menos conocidos que sus ventajas reales y potenciales. La adquisición de libros electrónicos en las bibliotecas universitarias se ve afectada por muchos factores, incluyendo las limitaciones de acceso, los formatos y las plataformas discutidos anteriormente. Algunos otros problemas, como su contenido, las características de los libros electrónicos y los modelos de acceso surgen de los propios libros y de sus proveedores. Otras limitaciones reflejan las necesidades y preferencias de los usuarios y de las instituciones. Las demandas de ciertas disciplinas o el actual desarrollo de la formación en línea pueden afectar en gran medida a las decisiones relacionadas con la adquisición de libros electrónicos (Blummer y Kenton, 2012). Conclusiones similares fueron esbozadas ya por Vasileiou y otros (2012b), en su informe de investigación que examinaba las percepciones y predicciones de los bibliotecarios académicos con respecto al papel futuro y al desarrollo de los libros electrónicos. Un punto muy interesante a destacar es que, según ellos, se trata de un proceso de dos vías en el que las partes interesadas en la adquisición de libros electrónicos no influyen solo directamente en las decisiones de las bibliotecas universitarias sobre el desarrollo de colecciones de libros electrónicos, sino que a su vez se ven afectados por esas decisiones. Las principales fuentes de adquisición de libros electrónicos en las bibliotecas universitarias son: 1) los editores que suministran libros electrónicos directamente a las bibliotecas; 2) los vendedores que ofrecen contenidos de diferentes editoriales y de acceso a los libros electrónicos a través de los sitios web de las editoriales, y 3) los agregadores que suministran contenido de diferentes editores en sus propias plataformas (Devenney y Chowcat, 2013). 
Hay muchas consideraciones y criterios importantes a tener en cuenta para la selección y adquisición de libros electrónicos en las bibliotecas universitarias. Las barreras para el acceso y las limitaciones en el uso tienen que ser consideradas entre ellas, pero, con base en los resultados del reciente estudio de Vasileiou et al., (2012a) se sugiere que los criterios clave de selección por parte de las bibliotecas universitarias son el coste de los libros electrónicos, su alto nivel de utilización y la demanda por parte de los usuarios de la biblioteca, las licencias, los modelos de negocio, las plataformas de acceso, las interfaces, la cobertura temática y el acierto en la elaboración de listas de lectura recomendadas según los estudios de cada universidad. Sin embargo, los bibliotecarios universitarios tienen también en cuenta el presupuesto de la biblioteca y las políticas de desarrollo de colecciones, así como las muchas restricciones que el uso de varias licencias traen consigo. Algunas de estas cuestiones se analizan con más detalle en los párrafos siguientes.

\subsection{Presupuestos y el precio del libro electrónico}

La diferencia entre considerar el precio de un libro impreso y el de un libro electrónico consiste en considerar a este último no sólo como un único costo de compra, sino también la cuestión de la financiación de las tarifas de acceso anuales (Bennett y Landoni, 2005). Además, hay diferentes maneras de adquirir libros electrónicos que deben compararse en términos de precio y costo. Así, no hay duda de que el presupuesto juega un papel importante en la construcción de la colección de libros electrónicos de la biblioteca universitaria. Ciertamente, puede ser una tarea muy difícil para las bibliotecas decidir cómo asignar los recursos financieros si la nueva colección tiene que ser financiada con cargo al presupuesto de la biblioteca existente y la forma de proceder a su actualización. Además, Tedd y Carin (2012) descubrieron que los bibliotecarios con presupuestos limitados son muy cautelosos a la hora de adquirir libros electrónicos cuando no saben que van a ser utilizados. Además, Burnette argumenta que el presupuesto es algo más que va más allá de los números:

"cuando se aplica a los recursos electrónicos, el objetivo del presupuesto, más que establecer un umbral de gasto o porcentajes es el establecer un acuerdo sobre la ayuda concedida para las colecciones digitales dentro de un año fiscal. También es un acuerdo filosófico de utilizar tecnología, personal y la información necesaria para facilitar ese acceso. El presupuesto es el punto de partida de un plan para acceder con éxito a las colecciones digitales". (Burnette, 2008, p. 4).

Los últimos años han visto una disminución significativa de los presupuestos de las bibliotecas universitarias y una proporción decreciente en la adquisición de libros, aunque también han sido años de un aumento de las asignaciones presupuestarias a recursos electrónicos. Muchas bibliotecas han asignado más de la mitad de sus presupuestos a contenidos electrónicos, si bien la mayor parte de esa cantidad está destinada a las revistas electrónicas (Blummer y Kenton, 2012), (Minčić-Obradovic, 2011) y (Vasileiou et al., 2012c). Schell sugiere que las bibliotecas universitarias, para comenzar a desarrollar su colección de libros electrónicos, deberían dedicar al menos el 10\% de su presupuesto total, previendo un aumento de hasta un $25 \%$ por la demanda de los usuarios (Schell, 2010). A pesar de que el gasto presupuestario en los libros electrónicos es cada vez mayor, las bibliotecas universitarias no parecen tener un presupuesto separado para su adquisición, sino que siguen adquiriendo títulos o colecciones de libros electrónicos dentro del presupuesto de compras general (Vasileiou et al., 2012c).

Diversos estudios han demostrado la importancia que posee el régimen de precios de los libros electrónicos sobre los bibliotecarios a la hora de tomar decisiones para su adquisición y explican la reticencia de las bibliotecas universitarias a invertir en ellos (Blummer y Kenton, 2012), (Mulvihill, 2011), (Vasileiou et al., 2012a) y (Vasileiou et al., 2012b). Lippincott et al. (2012) subrayan la importancia de los modelos de fijación de precios sostenibles y asequibles para los libros destinados a bibliotecas universitarias que los bibliotecarios tienen que desarrollar por su cuenta a partir de las oportunidades disponibles en el mercado. Los autores sugieren que para lograrlo:

"deben examinar críticamente los costos de las compras en una sola institución y compartir entre las instituciones y considerar cómo opciones existentes -como los multiplicadores sobre el precio de lista para una compra consorciada, costos de multiusuarios y de un solo usuario, precios diferenciados, y aprobar planes de descuentos- puede evolucionar en el beneficio mutuo de los bibliotecarios, editores y vendedores". (Lippincott et al., 2012, p. 6).

A pesar de que muchos creen que los libros de texto electrónicos son la solución a la asequibilidad y accesibilidad de los libros de texto en los campus universitarios, las ediciones digitales pueden ser tan costosas como las impresas y también pueden afectar a la accesibilidad para las personas con diversas discapacidades. Además, optar sólo por los libros electrónicos a su vez daría lugar a algunos problemas legales y educativos en las instituciones (Nelson y Hains, 2010). Según estudios recientes, los libros electrónicos a menudo cuestan más que sus contrapartes impresas, cuando los libros electrónicos académicos se compran individualmente, su precio puede ser incluso un $50 \%$ más alto que sus equivalentes impresos (Walters, 2012). También requieren costos adicionales para abonar el alojamiento en línea del 
contenido digital, los servidores y el mantenimiento de la red, el reformateo para múltiples plataformas de los formatos de archivo, así como proporcionar atención al cliente y el soporte técnico (Vasileiou et al., 2012a) y (Walters, 2013b). Este último autor sostiene que, incluso si los libros electrónicos fueran menos caros de producir, no se reducirían necesariamente los precios pagados por las bibliotecas.

\subsection{Los modelos de negocio}

Una serie de modelos de negocio están a disposición de las bibliotecas universitarias en todo lo relacionado con la adquisición de libros electrónicos para sus colecciones. Estos modelos varían de un proveedor a otro. Esta variedad sugeriría que las bibliotecas universitarias están bien atendidas por los proveedores de libros electrónicos, pero según Vassileiou et al.:

"es un hecho presente en la literatura especializada que la variedad de modelos de negocio de libros electrónicos proporcionada por los distribuidores resulta un problema para los bibliotecarios que se encuentran ante ellos una realidad complicada". (Vasileiou et al., 2012a, p. 23).

El surgimiento de nuevos modelos de compra podría incluso empeorar la situación, a pesar de que los editores están tratando de ayudar ofertando opciones adecuadas para diferentes bibliotecas. Según Minčić-Obradović (2011) la elección de un modelo depende principalmente de las necesidades de contenido de las instituciones. Vamos a tratar de introducir aquí una visión general de los modelos de negocio que están en uso por la mayoría de las bibliotecas, destacando sus ventajas e inconvenientes.

\subsubsection{Suscripción y alquiler}

El modelo de suscripción requiere el pago de una cuota (generalmente anual) para el acceso a una colección o a las materias de una colección ofrecida por un proveedor o un agregador. También puede consistir en un pago por visión (lectura en ese caso), además de una pequeña tarifa que permita el acceso inicial a toda la colección. Esta alternativa se considera también como un modelo de alquiler que permite el acceso a corto plazo a títulos individuales y se utiliza a veces como una alternativa a los préstamos interbibliotecarios. El modelo de suscripción es similar a las ofertas de las revistas electrónicas y se puede negociar a través de consorcios de bibliotecas, sobre todo, si ambos servicios son ofrecidos por el mismo agente. La suscripción es una de las formas más baratas de obtener acceso a grandes colecciones de libros electrónicos académicos. Sin embargo, no siempre es el modelo más atractivo especialmente cuando los bibliotecarios no tienen la posibilidad de decidir lo que debe estar disponible en el paquete y se puedan incluir materiales que no cumplan con las necesidades de una institución de educación superior. Además, "muchos vendedores y editores se reservan el derecho de añadir o retirar títulos de la colección durante el periodo de suscripción" (Walters 2013A, p. 196).

\subsubsection{Adquisición en función de un patrón y la selección basada en la evidencia}

La adquisición de libros en función de un patrón parece ser uno de los modelos de compra más discutidos en la literatura reciente. También está incluida entre las diez principales tendencias en las bibliotecas universitarias, identificándose este modelo como inevitable para bibliotecas bajo presión para demostrar que sus gastos están en línea con su valor. Este modelo cuenta con varias modificaciones, pero todos ellas emplean el mismo acceso a un cierto número de libros que se compran después del primer uso o son prestados a la biblioteca tras abonar una cierta suma de dinero. Los criterios se establecen en el inicio de un proyecto, la mayoría de los proveedores internacionales ofrecen algún tipo de modelo, requiriendo a una biblioteca seleccionar un conjunto de títulos que coincidan con ciertos criterios (por ejemplo, materia, editorial, precio) de un proveedor o de la colección de un agregador. Los registros bibliográficos para los títulos seleccionados se cargan en el catálogo para que puedan ser descubiertos por los usuarios. El primer uso de un título resulta en un arrendamiento a corto tiempo del título y desencadena el pago de una pequeña suma de común acuerdo entre la biblioteca y el proveedor de libros electrónicos (por ejemplo, un determinado porcentaje del precio de libro). La segunda (o tercera) vez que se usa el mismo título implicará la compra del mismo por parte de la biblioteca, quedando en propiedad. La primera vez que se accede a un título, la biblioteca recibe notificación de ello y puede retirarlo de la colección. Nuevos títulos pueden ser añadidos al corpus periódicamente (Medeiros, 2011, p. 160). Este modelo permite a una biblioteca adquirir libros electrónicos solicitados por sus usuarios. Los estudios muestran mayores tasas de circulación de los bienes adquiridos en este modelo que los adquiridos por las decisiones de la biblioteca (Tyler, Falci, Melvin, EPP y Kreps, 2013).

Por otro lado, Fischer, Wright, Clatanoff, Barton y Shreeves realizan una pregunta válida respecto de los presupuestos de las bibliotecas y la adquisición según un patrón de elaboración de presupuestos responsable, acerca de las decisiones de selección realizadas por un subconjunto no identificado de un alto número de usuarios potenciales (Fischer et al., 2012, p 490). Una de las principales preocupaciones de los bibliotecarios académicos relacionadas con este modelo es el miedo a la construcción de una colección desequilibrada y de menor calidad (Bucknell, 2012; Fischer et al., 2012; Walters, 2012). 
La selección basada en la evidencia es uno de los modelos más nuevos de compra y, comparado con la adquisición en función de un patrón, es escasamente discutido hasta ahora por los autores especializados. Bucknell ofrece una explicación detallada de cómo funciona este modelo:

"bajo este modelo, la biblioteca paga una cuota por adelantado relativamente modesta con el fin de ser capaz de acceder a una colección por un año. Hacia el final de ese año, la biblioteca puede evaluar sus informes de uso para decidir qué títulos o colecciones son las que debe retener en acceso permanente, computando el coste y teniendo en cuenta el pago efectuado previamente. Llegados a ese punto, el acceso a los títulos no retenidos se pierde a menos que la biblioteca y el vendedor estén de acuerdo en prolongar un año más la adquisición basada en evidencia". (Bucknel, 2012, p. 58).

Este autor sostiene que este modelo es beneficioso tanto para las bibliotecas como para los proveedores. Para las bibliotecas, reduce el riesgo de comprar un paquete que resulte ser poco utilizado y les permite evaluar su estrategia de compra. Los vendedores pueden verse atraídos por este modelo porque proporciona un nivel mínimo garantizado de ingresos y también puede conducir a una compra más grande, algo que una biblioteca no habría estado nunca dispuesta a hacer sin disponer de una evidencia de uso.

\subsubsection{Compra de títulos individuales y colecciones en titularidad perpetua}

Vassileiou et al. (2012a) señalan que el modelo de negocio más popular entre los bibliotecarios es la adquisición de libros de forma permanente. Este modelo permite a una biblioteca comprar un título individual o colecciones de libros electrónicos directamente desde un editor mediante el pago por adelantado (incluso hasta un par de años). Tener un acceso permanente a los libros electrónicos aún requiere el pago de una cuota de mantenimiento para el alojamiento permanente del material comprado en la plataforma de un editor (Morris y Sibert, 2010) y (Vasileiou et al., 2012a).

Las bibliotecas universitarias tienen, por lo general, posibilidad de adquirir libros electrónicos como títulos individuales o bien paquetes de libros electrónicos. En ambos casos, la propiedad permanente significaría la eliminación de la mayoría de las restricciones de gestión de derechos digitales en el uso de los artículos comprados. Sin embargo, a veces, un sistema de crédito se adjunta a los artículos comprados permitiendo prestar el libro un cierto número de veces durante un período determinado de tiempo, como por ejemplo un año. Cuando se consumen estos créditos, una biblioteca puede comprar otra copia o esperar el nuevo período en que los créditos se restauren a la copia ya comprada.

Uno de los inconvenientes de la adquisición de los libros electrónicos como paquetes es que los bibliotecarios académicos rara vez pueden elegir qué títulos se incluyen en los paquetes. En el lado positivo, los paquetes de libros electrónicos se ofrecen a un precio mucho más atractivo que el precio de los títulos individuales y también ahorran tiempo en la selección, adquisición y procesamiento (Ashcroft, 2011).

La adquisición de los libros electrónicos como títulos individuales consume mucho tiempo, el bibliotecario tiene que averiguar si un título está disponible como libro electrónico, en qué plataforma o plataformas está disponible, y cuánto cuesta según el modelo de negocio. Según Vasileiou et al. (2012a, p. 25): "la selección título por título facilita un mejor uso de los fondos y proporciona el máximo control sobre las decisiones de adquisición, previniendo la compra de título de menor calidad o fuera del ámbito de interés de la biblioteca". Mulvihill afirma que "la mayoría de los bibliotecarios quieren que el libro electrónico se comporte como los libros impresos, que son relativamente fáciles de leer y comprar" y ofrecerán las mismas ventajas para el desarrollo de las colecciones (Mulvihill, 2011, p. 13). Esta declaración explica la tendencia de los bibliotecarios de adquirir libros electrónicos en propiedad permanente.

Por lo tanto, ninguno de los modelos de negocio existentes para la adquisición de libros electrónicos es el ideal para las bibliotecas universitarias y, en todo caso, el poder sobre el uso y manejo de los libros recae en el editor. En todos los casos, incluso cuando los libros se compran con carácter permanente, subsiste la amenaza de perder el acceso a los libros electrónicos porque la colección es abandonada por su titular (un editor, un proveedor o un agregador) o porque cambian las condiciones de acceso. No hay soluciones de preservación digital permanentes incorporadas en las plataformas de los editores, lo que se traduce en un problema adicional en el manejo de estos recursos por parte de las bibliotecas.

\subsection{Licencias de libros electrónicos}

Uno de los principales factores que cambian la manera en la que los bibliotecarios trabajan con los libros electrónicos en contraposición a cuando lo hacen con libros impresos, es que los electrónicos son controlados no solamente por una ley de derechos de autor, sino que requieren la aplicación de un derecho contractual a través de licencias (Müller, 
2012). Estas licencias representan una enorme carga para las bibliotecas universitarias. El problema está bien resumido por la Comisión Europea:

"las bibliotecas y universidades subrayan la complejidad y fragmentación del sistema actual de acuerdos de licencias con los editores. Una universidad europea típica es requerida para firmar un centenar o más de licencias que rigen el uso de material digital de investigación suministrado por varias editoriales. Examinar lo que cada una de estas licencias individuales permite con respecto a, por ejemplo, el acceso, la impresión, el almacenamiento y el derecho de autor es un proceso engorroso”. (Müller, 2012, p. 153).

Dado que la mayoría de los libros electrónicos de las bibliotecas universitarias son alquilados y no comprados, las bibliotecas deben reajustar sus esquemas de funcionamiento y percibir que ya no son dueños de la colección, e incluso más, dependiendo de la licencia, las bibliotecas todavía podrían tener que abonar pagos recurrentes para el acceso a un conjunto de títulos que no mejora con el tiempo (Walters, 2013b). Los editores y agregadores suelen tener una gran variedad de modelos de licencias que controlan cómo los usuarios pueden tener acceso a los libros electrónicos. Por otra parte, Walters (2012) sostiene que la mayoría de los vendedores de libros electrónicos han adoptado modelos de licencias que impiden a los usuarios tomar ventaja de los beneficios que la tecnología del libro electrónico podría proporcionar. Por lo tanto, son muchas las consideraciones que las bibliotecas universitarias tienen que tener en cuenta con respecto a las licencias de los libros electrónicos. Los bibliotecarios necesitan familiarizarse con estos problemas de licencias y su terminología, para ser capaces de identificar las limitaciones y restricciones inherentes a los acuerdos de licencia (Vassileiou et al., 2012a). La negociación de los términos de las licencias a través de consorcios podría ser una opción interesante para las bibliotecas universitarias. Un reciente estudio (Vasileiou et al., 2012c) sugiere que un equipo experimentado puede conseguir mejores ofertas, reducir los múltiples esfuerzos de negociar por separado con cada editor y obtener descuentos en las tarifas de alojamiento en las plataformas de acceso. Por otra parte, a través de la compartición de libros electrónicos dentro de un consorcio de bibliotecas, se reduce también el esfuerzo añadido de la formación del personal local en estas nuevas habilidades y también se reduce el trabajo que supone la catalogación, ya que ambas tareas se llevarán a cabo de forma centralizada por el consorcio (Schell, 2010).

La definición de un usuario autorizado, las restricciones del préstamo interbibliotecario, la aplicación de un uso justo, el intercambio académico, los problemas de autenticación IP y la incorporación de libros electrónicos dentro de los entornos virtuales de aprendizaje de las universidades son sólo algunos de los muchos otros temas a considerar (Stamison, 2011) y (Vasileiou et al., 2012a). Desde las bibliotecas adquieren libros electrónicos a un número diferente de proveedores, esto significa que tienen que hacer frente a los diferentes modelos de licencia y con diferentes términos. Tedd (2005) hace hincapié en la importancia de hacer que los usuarios tomen conciencia de estas diferencias, ya que serán ellos quienes sientan sus efectos. Thompson y Sharp (2009) llevaron a cabo una sesión con personal de la biblioteca que reveló que muy pocos bibliotecarios sintieron que su biblioteca estaba mejorando en este campo.

Resumiendo brevemente la primera parte de este trabajo, es posible argumentar que, aunque los principios básicos de la adquisición no han cambiado con la llegada de los libros electrónicos, las condiciones de su compra, mantenimiento, acceso y uso por parte de las bibliotecas y sus usuarios pueden llegar a ser bastante complicadas, aunque las ventajas parecen ser más atractivas que el temor a las complicaciones.

\section{LIBROS ELECTRÓNICOS EN LAS BIBLIOTECAS UNIVERSITARIAS DE SUECIA}

Bastante se ha escrito sobre libros electrónicos en las bibliotecas suecas y aún prosigue un apasionado debate público sobre la cuestión de su disponibilidad y de los modelos de negocio para el acceso. La mayor parte de la discusión se refiere a las bibliotecas públicas y al mercado de consumo masivo, los aspectos relacionados con las bibliotecas universitarias no se consideran muy a menudo. Las diferencias entre el mercado del libro académico (de investigación), profesional (técnico) y material docente en todo lo relacionado con el libro electrónico, sólo se mencionan fugazmente en los informes oficiales elaborados por el gobierno sueco (Suecia. Ministerio de Cultura, 2012, p. 228) y el parlamento sueco (Suecia. Parlamento, 2013/14: RFR3, p. 14). Esto se explica, en parte, por Svedjedal (2012) quien en su informe a la Asociación Sueca de Editores en un breve capítulo (tres páginas) explica el entorno de las editoriales académicas y las bibliotecas universitarias, señalando el predominio de los proveedores internacionales de recursos digitales en estas bibliotecas y las realidades de la publicación electrónica en el ámbito de la comunicación académica (revistas electrónicas y monografías electrónicas). En 2011, la Biblioteca Nacional de Suecia presentó un informe mapeando los problemas relacionados con la introducción de los libros electrónicos en las actividades de las bibliotecas del país (Kungliga Biblioteket, 2011). Este informe indicó que no existía ningún problema con la adquisición y el suministro de libros electrónicos en las bibliotecas universitarias: por entonces la mayoría de las bibliotecas universitarias en Suecia ya había aceptado la política de que, si hay que elegir entre un libro impreso y un libro electrónico, se adquiriría el libro electrónico, especialmente aquellos libros utilizados en la 
enseñanza (Gustaf, 2010). Parece que la principal línea de investigación en temas de presencia de libros electrónicos en las bibliotecas universitarias suecas se lleva a cabo por los estudiantes que escriben sus trabajos fin de grado en los departamentos de Biblioteconomía y Ciencias de la Información, algo que parece lógico. Algunos bibliotecarios también están llevando a cabo pequeñas investigaciones en el seno de sus lugares de trabajo. Byström (2012) ha comparado las estadísticas de diferentes paquetes de libros electrónicos proporcionados por distintos proveedores en la Biblioteca de la Universidad de Uppsala y llegó a la conclusión de que son engañosas e incomparables. Según esta autora, la falta de estadísticas comparables no permite a los bibliotecarios responsables de la adquisición de fondos disponer de datos fiables para tomar decisiones acertadas en el proceso de acceso a la renovación de la suscripción, lo que hace imposible justificar debidamente las compras o no permite evaluar el costo-beneficio del uso de los libros electrónicos (Byström, 2012, p. 220).

El artículo de Söderback (2011) sobre la situación en las bibliotecas universitarias suecas discute la infraestructura para la gestión de los libros electrónicos, a saber, el papel de la herramienta de resolución de hipervínculos $\mathrm{SFX}^{4}$ comprada por un consorcio de bibliotecas universitarias suecas (BIBSAM) en el año 2004 y su uso dentro del catálogo colectivo. Este artículo también menciona que el consorcio compra revistas electrónicas para las bibliotecas suecas, pero deja la gestión de los libros electrónicos a las bibliotecas locales. Las estadísticas del año 2009 mostraban que se descargaron 5.598.777 libros electrónicos desde las universidades o colegios universitarios. El autor también señala que:

"las discusiones con las bibliotecas sugieren que los libros electrónicos no precisan más del 6\% del dinero gastado en adquisiciones. Bajo esta perspectiva, los libros electrónicos son muy utilizados y parecen ser una buena inversión para las bibliotecas universitarias". (Söderback, 2011, p. 38).

A pesar de esto, los libros electrónicos siguen siendo una proporción muy pequeña en comparación con las colecciones de libros impresos en las bibliotecas universitarias suecas. Pero si nos fijamos en los libros recién comprados en el año 2012, vemos que la situación es totalmente diferente en todo el país (ver Figura 1) y en las universidades concretamente: un 70\% (global) frente al 88\% (en algunas de las universidades) de todas las nuevos adquisiciones son libros electrónicos (Maceviciute y Borg, 2013, p. 16).

\section{Book stock (printed, e-books) Acquisition (printed, e-books)}

\section{Swedish universities 2012 (Fron Borg. Martin2013, based on Swedish staistics 2012)}
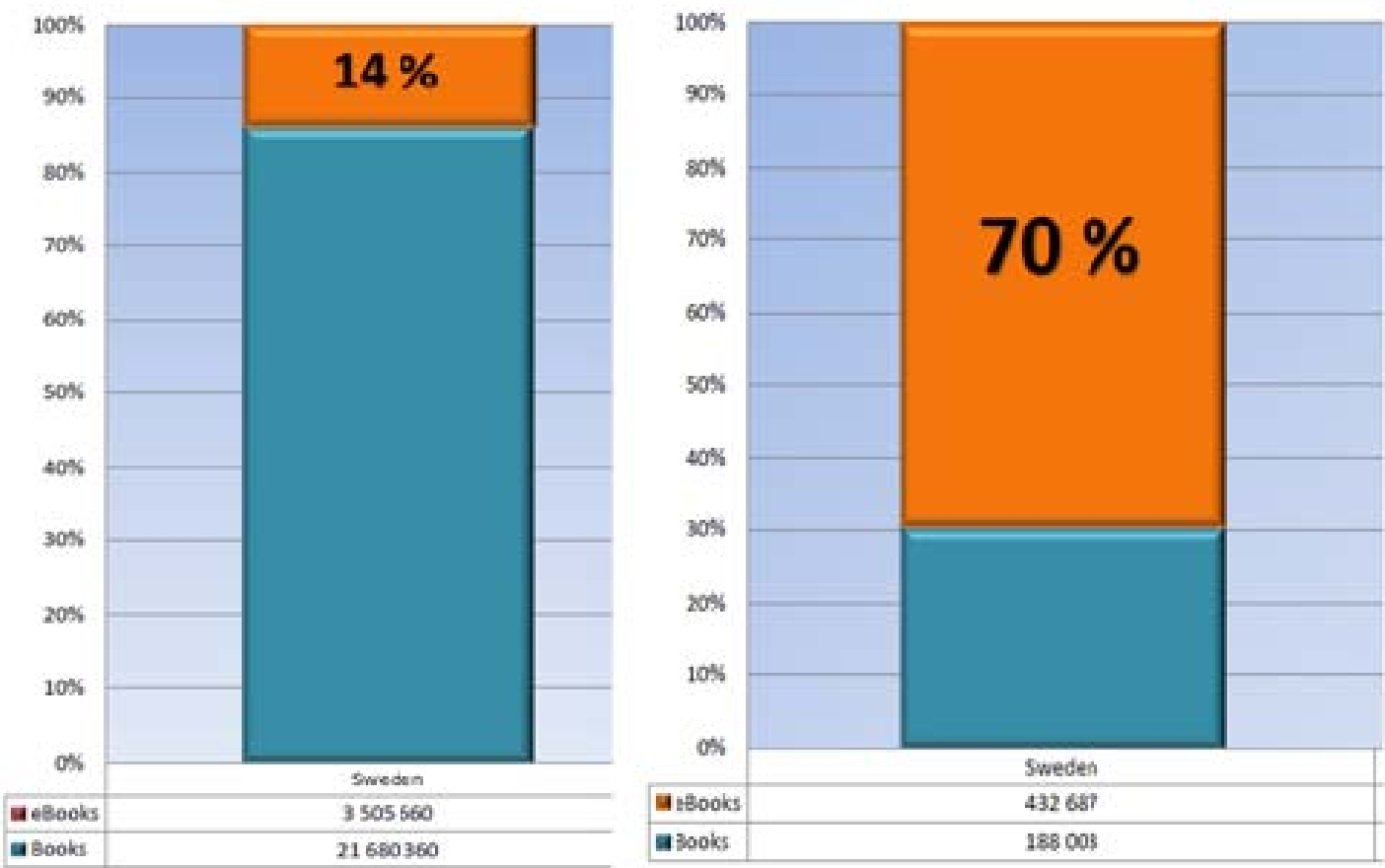

Figura 1. Libros electrónicos en las colecciones de bibliotecas universitarias y adquisición en 2012. (Fuente: Maceviciute y Borg, 2013).

Esta tendencia también coincide con las inversiones que las bibliotecas universitarias suecas han hecho recientemente de acuerdo con la política aceptada de invertir cada vez más en publicaciones editadas en este formato. 
A continuación vamos a ilustrar los temas de adquisiciones de libros electrónicos en las bibliotecas universitarias suecas mediante la presentación de dos breves estudios de caso que, en principio, deberían ser diferentes ya que uno representa una universidad de tamaño medio, con un menor número de estudiantes y de personal y con una estructura de gestión relativamente sencilla, mientras que el otro analiza una antigua gran universidad de investigación con muchos empleados de diferente tipo, un gran número de estudiantes y con estructuras complejas de gestión en las unidades académicas y de su biblioteca.

\subsection{Dos estudios de caso: una regional de tamaño medio y una gran universidad de investigación}

\subsubsection{Estudio de caso 1}

La universidad regional del primer caso de estudio (en lo sucesivo, U1) hizo recientemente una inversión muy grande para adquirir libros electrónicos. La biblioteca U1 está trabajando bajo la creciente presión de unos presupuestos decrecientes, de hecho, en el año 2013 el presupuesto fue severamente escaso en comparación con años anteriores. Los objetivos de la biblioteca son para apoyar los objetivos principales de la universidad en el aumento de la financiación externa para la investigación, apoyar el aprendizaje de los estudiantes y ayudar en las relaciones con empresas e instituciones del mundo laboral asociadas a la universidad a través de la provisión de recursos de información, consultoría y capacitación.

En la actualidad, la colección de libros electrónicos de la biblioteca U1 es tan grande como la de materiales impresos (108.456 frente a 114.313), aunque el ritmo de adquisición de libros electrónicos es mucho mayor que el de impresos. El nivel de uso y el volumen de préstamos de los libros electrónicos superan también a los de libros impresos. Con respecto a la política de adquisiciones aprobada por la biblioteca U1, la preferencia en la adquisición se confiere a un libro electrónico, si es que existe. El ochenta por ciento de todo el presupuesto de los medios de comunicación va a material electrónico: libros, revistas y bases de datos. Esta biblioteca ha decidido estar abierta a la mayoría de los modelos de negocio de adquisición de libros electrónicos durante esta primera fase de desarrollo de las colecciones de libros electrónicos. Se espera poder elegir uno o varios modelos más apropiados más adelante.

\subsubsection{Estudio de caso 2}

Una universidad de investigación es la analizada en este segundo estudio (en lo sucesivo, U2). Esta institución tiene una larga historia y gran tradición, siendo una de las universidades de investigación suecas mejor clasificada en los rankings. Además posee una fuerte reputación internacional. Su complejidad estructural y de gestión se refleja en la complejidad de su biblioteca (biblioteca U2) que consta de varios grandes departamentos, incluyendo más de veinte unidades organizativas, la mayoría de las cuales también funcionan como bibliotecas autónomas. La colección de la biblioteca contiene millones de artículos (impresos y digitales). La biblioteca tiene acceso a aproximadamente 400.000 libros electrónicos, que es sólo una pequeña parte de su colección general de libros impresos y también una pequeña parte de sus recursos digitales, donde predominan las revistas a texto completo y bases de datos bibliográficas. Esta biblioteca experimenta problemas de comunicación con el personal universitario, la misma resulta enormemente dispersa, especialmente hacia los investigadores, quienes prefieren el uso de recursos de la biblioteca de forma remota. Esta biblioteca mantiene más contacto con los estudiantes universitarios tanto en la biblioteca principal como en las bibliotecas sucursales.

\subsection{Análisis comparativo de las dos bibliotecas que conforman los estudios de caso}

\begin{tabular}{|c|c|}
\hline $\begin{array}{l}\text { U1 biblioteca } \\
\text { Política de adquisiciones }\end{array}$ & biblioteca \\
\hline $\begin{array}{l}\text { - Los libros electrónicos están incluidos en } \\
\text { la política general. Se le dio prioridad a } \\
\text { su adquisición para satisfacer las } \\
\text { expectativas de los estudiantes en } \\
\text { obtener acceso a los recursos digitales. } \\
\text { - Los libros electrónicos se adquieren } \\
\text { comercialmente. } \\
\text { Otros tipos de documentos digitales } \\
\text { (libros antiguos digitalizados por la } \\
\text { biblioteca u otros agentes) no se } \\
\text { consideran libros electrónicos. }\end{array}$ & $\begin{array}{l}\text { - La adquisición de libros electrónicos no se menciona } \\
\text { como algo específico dentro la política general, pero } \\
\text { los recursos digitales tienen preferencia en la } \\
\text { adquisición. } \\
\text { Los libros electrónicos se caracterizan } \\
\text { principalmente como objetos digitales comprados y } \\
\text { son usados de manera similar a los libros impresos y } \\
\text { con el mismo propósito. } \\
\text { Hay una clara distinción entre los libros electrónicos } \\
\text { y otros materiales digitales. }\end{array}$ \\
\hline
\end{tabular}




\begin{tabular}{|c|c|}
\hline lentes & \\
\hline 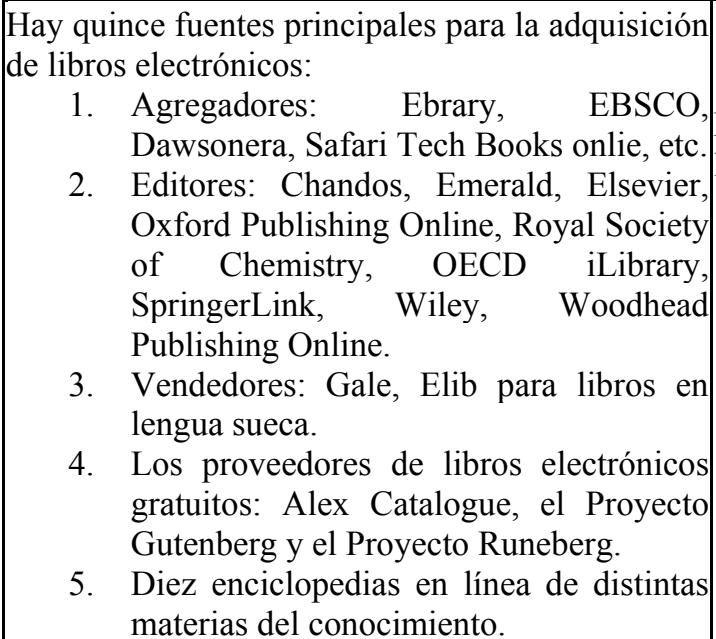 & $\begin{array}{l}\text { Hay treinta y ocho fuentes principales para la adquisición de } \\
\text { libros electrónicos: } \\
\text { 1. Agregadores: DawsonEra, Ebrary, EBSCO Knovel, } \\
\text { OECD, Safari. } \\
\text { 2. Editores: ASTM, Cambridge Onine, Emerald, } \\
\text { Elsevier, Grypho Ediciones, IEEE, FMI, McGraw } \\
\text { Hill, MedicinesComplete, Morgan and Claypool, } \\
\text { Oxford Online, Sage, Spie, Springer, Thieme, World } \\
\text { Bank. } \\
\text { 3. Vendedores: libros europeos tempranos, EECO, } \\
\text { EEBO, Gale, PsycBOOKS. }\end{array}$ \\
\hline Tamaño y estructura de la colección & \\
\hline $\begin{array}{ll}\text { - } & \text { Materiales impresos: } 114313 \\
\text { - } & \text { Revistas electrónicas: } 36.900 \\
\text { - } & \text { Libros electrónicos: } 108456 \\
\text { - } & \text { Bases de datos (comerciales): } 86 \\
\text { - } & \text { Préstamos interbibliotecarios: } 1249\end{array}$ & $\begin{array}{l}\text { - } \text { Materiales impresos: "varios millones". } \\
\text { - Revistas electrónicas: sin datos. } \\
\text { - Libros electrónicos: } 400.000 \\
\text { - Bases de datos: más de } 500 \text { (incluyendo } \\
\text { bibliográficas, factuales, a texto completo, libros } \\
\text { electrónicos enciclopédicos, etc.). }\end{array}$ \\
\hline Los modelos de negocio & \\
\hline $\begin{array}{l}\text { Suscripción: La colección Ebrary Academic } \\
\text { completa, con más de } 80.000 \text { títulos y Safari Tech } \\
\text { Books online con casi } 7.000 \text { títulos. } \\
\text { Ventajas: son relativamente baratos y permite } \\
\text { acceso a un gran número de artículos. La mayoría } \\
\text { de los libros se pueden descargar para lectura } \\
\text { fuera de línea en lectores electrónicos, } \\
\text { ordenadores o tabletas. } \\
\text { Deficiencias: los sistemas DRM imponen límites } \\
\text { a la impresión y a la copia de los materiales } \\
\text { debido a los derechos de autor. También influyen } \\
\text { en los tiempos de préstamo, existen limitaciones } \\
\text { en el número de días que el lector puede tener un } \\
\text { libro. Los libros pueden desaparecer de las } \\
\text { colecciones sin notificación previa. }\end{array}$ & $\begin{array}{l}\text { Suscripción: La biblioteca U2 mantiene un importante } \\
\text { número de suscripciones a paquetes a través del servicio } \\
\text { central de la biblioteca. } \\
\text { Ventajas: un gran número de libros académicos de alta } \\
\text { calidad a disposición de los usuarios. Muchos paquetes } \\
\text { ofrecidos por diferentes vendedores son adecuados para } \\
\text { diferentes disciplinas e investigadores. } \\
\text { Deficiencias: cada paquete viene con diferentes reglas y } \\
\text { condiciones de uso que se debe comunicar a los usuarios. }\end{array}$ \\
\hline
\end{tabular}




\begin{tabular}{|c|c|}
\hline $\begin{array}{l}\text { Adquisición: La biblioteca da una suma fija de } \\
\text { dinero al proveedor Ebrary y selecciona una serie } \\
\text { de colecciones de libros electrónicos. El sistema } \\
\text { está configurado para préstamos de corta } \\
\text { duración. Cuando el libro se utiliza por segunda } \\
\text { ocasión, es comprado por la biblioteca. Cuando un } \\
\text { libro es utilizado por primera vez, la biblioteca } \\
\text { recibe un mensaje y puede decidir si ese libro|t } \\
\text { debe permanecer en la colección. } \\
\text { Ventajas: El primer uso es barato (10-15\% del } \\
\text { precio de la concesión de la licencia) y mientras } \\
\text { que el uso sea del } 2 \% \text { de la recaudación Ebrary } \\
\text { (3000 libros) todo parece estar bien. Los libros se } \\
\text { compran a propósito y por necesidad existente. } \\
\text { Para la adquisición basada en la evidencia } \\
\text { proporcionada por Elsevier no hay necesidad de } \\
\text { tomar una decisión sobre la compra y la selección } \\
\text { es posible después de evaluar los datos de uso de } \\
\text { los títulos. } \\
\text { Deficiencias: El primer uso es por siete días } \\
\text { solamente. Cuando el uso se incrementa, aumenta } \\
\text { el gasto y resulta imposible planificar el } \\
\text { presupuesto. Requiere un monitoreo constante de } \\
\text { la situación con el acceso a los libros electrónicos } \\
\text { en la colección. En el año } 2014 \text { el presupuesto } \\
\text { para la adquisición en función de un patrón se } \\
\text { consumió en un mes. La adquisición basada en } \\
\text { evidencias no tiene este inconveniente. }\end{array}$ & $\begin{array}{l}\text { Adquisición: Los bibliotecarios tienen en cuenta las } \\
\text { sugerencias y peticiones del personal y de los estudiantes y } \\
\text { tratan de adquirir los libros solicitados, pero no tienen en } \\
\text { cuenta la compra porque sería demasiado caro. Tratan de } \\
\text { encontrar el título solicitado en paquetes ya adquiridos, } \\
\text { siguiendo la modalidad de compra en función de un patrón } \\
\text { establecido por la propia biblioteca en el año 2012. En este } \\
\text { contrato, están los tres primeros usos pagados en forma de } \\
\text { préstamo y, en el caso del cuarto uso, la biblioteca compra el } \\
\text { libro electrónico. } \\
\text { Ventajas: Se compra tras un uso real por parte de los usuarios. } \\
\text { Es una buena manera de comprar más libros y ahorrar } \\
\text { recursos. } \\
\text { Deficiencias: Resulta difícil de mantener este sistema en el } \\
\text { marco del presupuesto. Resulta impredecible en la calidad y } \\
\text { pertinencia de los títulos ofrecidos y seleccionados. La } \\
\text { selección puede ser sesgada si un usuario es particularmente } \\
\text { activo. }\end{array}$ \\
\hline $\begin{array}{l}\text { Compra eligiendo modelo: Compra a través de } \\
\text { agregadores como Ebrary, DawsonEra y Ebsco. } \\
\text { Ventajas: La biblioteca posee el libro y es } \\
\text { relativamente fácil satisfacer las solicitudes de } \\
\text { compra. Para un número ilimitado de usuarios es } \\
\text { la mejor opción. DawsonEra introdujo un sistema } \\
\text { de créditos para determinar cuántas veces al año } \\
\text { un libro puede ser utilizado (125-400). Los libros } \\
\text { están disponibles para descargar y leer fuera de } \\
\text { línea. } \\
\text { Deficiencias: Las limitaciones en el número de } \\
\text { copias e impresiones. El número de lectores } \\
\text { simultáneos. El alto coste de los productos para un } \\
\text { número ilimitado de usuarios (1,5 veces más que } \\
\text { un libro impreso). Las limitaciones impuestas por } \\
\text { el sistema de crédito y el hecho de que estas } \\
\text { restricciones pueden cambiar en el caso de } \\
\text { artículos populares. }\end{array}$ & $\begin{array}{l}\text { La compra a los vendedores es responsabilidad de los } \\
\text { bibliotecarios de los departamentos. EBL y Dawsonera } \\
\text { dominan la lista de proveedores de libros electrónicos. } \\
\text { Ventajas: La compra a un número limitado de proveedores } \\
\text { mantiene bajo el número de plataformas de libros electrónicos } \\
\text { en uso. } \\
\text { Deficiencias: El vendedor establece las normas de acceso y } \\
\text { uso. Un bibliotecario no tiene oportunidad de elegir vendedor } \\
\text { por el mejor precio. Es imposible comprar libros suecos, } \\
\text { incluso si existen, a los proveedores internacionales. Los } \\
\text { libros electrónicos a menudo pueden ser mucho más caros que } \\
\text { las versiones impresas. Las cuestiones de precios resultan } \\
\text { finalmente la cuestión clave para tomar una decisión final } \\
\text { sobre qué comprar. Los títulos más nuevos no siempre están } \\
\text { disponibles como libros electrónicos. }\end{array}$ \\
\hline $\begin{array}{l}\text { Compra de libros electrónicos a editores. } \\
\text { Elsevier, Springer, Wiley-Blackwell, Woodhead. } \\
\text { Ventajas: Existen pequeñas limitaciones y } \\
\text { restricciones de uso. Se pueden negociar } \\
\text { descuentos para varias colecciones. } \\
\text { Deficiencias: Resulta más caros que comprar } \\
\text { libros impresos y las suscripciones a los libros } \\
\text { electrónicos. La compra individual de títulos es un } \\
\text { proceso muy lento para los bibliotecarios, } \\
\text { consume mucho esfuerzo. }\end{array}$ & $\begin{array}{l}\text { La compra a los editores es algo inusual en la biblioteca U2. } \\
\text { Ventajas: La biblioteca puede conseguir mejores sistemas } \\
\text { DRM (en términos de menores restricciones para utilizar los } \\
\text { libros electrónicos), eligiendo el editor más liberal (por } \\
\text { ejemplo, Springer). } \\
\text { Deficiencias: Diferentes editores tienen distintas plataformas } \\
\text { y la compra a muchos aumentaría la complejidad en el trabajo } \\
\text { de los bibliotecarios y para el acceso de los lectores. }\end{array}$ \\
\hline
\end{tabular}


Libros en sueco: sólo disponibles en el serviciolLibros en sueco: no están disponibles como libros Elib (propiedad de cuatro editoriales más electrónicos. Se calcula que aproximadamente el $98 \%$ de la grandes). Cada préstamo cuesta a la biblioteca 20 colección de libros electrónicos está en idiomas extranjeros, coronas $(\approx 2.25 €)$.

predominantemente en idioma inglés con algunas inclusiones Ventajas: Hay algunos libros de no ficción en de francés y noruego. Incluso la presencia de libros impresos sueco. Ayuda a promover la lectura de ocio a los suecos en algunas de las unidades de la biblioteca puede ser estudiantes y al personal. casi testimonial, dependiendo de la naturaleza de las Deficiencias: Resulta complicado planificar los disciplinas. Otras disciplinas (como Enfermería, por ejemplo) costes. La cantidad de títulos disponible de la sí acusan la experiencia de una severa falta de libros de texto colección de no-ficción es muy limitada. Los en idioma sueco en formato de libro electrónico. Los libros libros pueden dejar de estar en la colección, perosuecos se pueden comprar en Dawson o Adlibris cuando es se anuncia de antemano. necesario.

\section{Procesos de presupuesto y compra}

Presupuestos. El presupuesto es aprobado por la biblioteca de la universidad. Su director/a decide EI presupuesto para adquisiciones de la biblioteca es visto cómo se utilizarán los fondos, incluyendo la como excepcionalmente alto y muchos miembros de la gestión de la adquisición y la gestión de medios. universidad tienen dificultades para comprender este coste. El Las negociaciones son llevadas a cabo por rectorado asigna el presupuesto para la biblioteca dentro del personal bibliotecario especializado en libros presupuesto general de la universidad.

electrónicos con los representantes de los Las negociaciones son llevadas a cabo por equipos vendedores que suelen llegar a la biblioteca de la especializados en la adquisición de paquetes grandes de universidad tras el anuncio de una licitación recursos digitales. Los bibliotecarios sugieren buscar la pública.

"mejor gestión DRM", es decir, que las limitaciones al acceso

Las licencias y contratos son firmados por la al libro digital sean las menores posibles. La mayor parte de dirección de la biblioteca o por el rectorado (enlos recursos digitales se adquieren a través del consorcio los casos de grandes pagos). Las licencias son BIBSAM que auna a las bibliotecas suecas universitarias y de archivadas en el registro de la universidad. investigación.

\section{Consorcios}

El consorcio BIBSAM no puede firmar contratos con los agregadores, pero obtiene un buen precio de algunos editores que venden a través de consorcios (por ejemplo, Emerald y Royal Society of Chemistry), sobre todo cuando se compran las colecciones o las negociaciones incluyen otros La biblioteca se suscribe a la mayor parte de los recursos digitales adquiridos por el consorcio BIBSAM, incluyendo los paquetes de libros electrónicos.

productos.

\section{Plataformas y formatos}

Aproximadamente diez plataformas están en uso por biblioteca. Los formatos más utilizados son Cada colección tiene una plataforma y una interfaz diferente. Epub y PDF. Los distintos proveedores permiten a Incluso con la introducción de una sencilla función de los usuarios leer libros utilizando diferentes búsqueda en los catálogos, los usuarios se confunden por las sistemas: AdobeReader, Bluefire, etc. $\quad$ diferentes condiciones impuestas por los proveedores.

\section{Ventajas generales y beneficios de los libros electrónicos}

Para los lectores: Muchos lectores pueden utilizar el mismo texto al mismo tiempo. Los libros son accesibles desde cualquier lugar y en cualquier momento al mismo tiempo. Los lectores pueden Para los lectores: Se dispone de una gran cantidad de libros tener muchos libros sin necesidad de llevar a cabolacadémicos que son bastante nuevos y de alto nivel. Los una descarga y pueden leerlos en cualquier lugar. lectores pueden descargar los libros en cualquier lugar que Para las bibliotecas: Existe un gran ahorro en el deseen.

tiempo dedicado al procesamiento, catalogación y Para las bibliotecas: Los libros electrónicos no ocupan provisión del acceso al libro. Los libros espacio físico y dejan lugar para habilitar salas de lectura.

electrónicos no ocupan espacio físico. No hay necesidad de enviar paquetes de libros de una biblioteca a otra. 


\begin{abstract}
Barreras generales y los problemas de los libros electrónicos
Para los lectores: todas las limitaciones Para los lectores: el que existan diferentes plataformas para inherentes a los sistemas DRM. Los lectores cada colección detrae el uso del libro electrónico. Las tienen que llegar a conocer múltiples interfaces limitaciones inherentes de los sistemas DRM. Los usuarios no (aproximadamente diez) en distintas plataformas. están acostumbrados a utilizar los libros electrónicos y optan, El software de lectura es diferente para los libros a veces, por comprar ellos mismos un libro impreso si la electrónicos en cada proveedor.

biblioteca adquiere sólo una versión electrónica. Las Para las bibliotecas: Siempre es un editor quien pequeñas pantallas de los dispositivos de lectura y de los decide las condiciones de uso. La biblioteca noteléfonos móviles hacen que sea difícil leer libros tiene poder sobre ello y en algunos casos ha de electrónicos. El uso está restringido a los miembros de la imponer sus propias limitaciones para el acceso. universidad.

Encontrar libros electrónicos existentes, comparar Para las bibliotecas: Los bibliotecarios evalúan las múltiples su disponibilidad y precios, las condiciones, la plataformas y se convierten en imagen de marca ('branding') toma de decisiones de adquisición de un títulodentro de las campañas de marketing de los editores. Los seleccionado, etc. son tareas que conllevan muchoproveedores de libros electrónicos tienen todo el poder sobre tiempo. Incluso en el caso de una compra de unla configuración de las restricciones sobre el uso de los libros libro en propiedad, el libro sólo es accesible a electrónicos (número máximo de múltiples usuarios, través de la plataforma de un editor (aunque sedescargas, impresiones). La biblioteca no tiene ninguna eliminan las limitaciones). Los editores no influencia sobre estos criterios. Los usuarios tienen limitado permiten a las bibliotecas utilizar libros el conocimiento global de las características de la colección electrónicos para los préstamos de libros electrónicos y de las condiciones de su uso. Es interbibliotecarios, aunque el envío de losimposible evaluar si se utilizan los libros electrónicos y capítulos está permitido en muchos casos. DRM y cuánto a partir de estadísticas de uso. Las cambiantes otros sistemas limitan las posibilidades de uso de condiciones de uso para los títulos adquiridos, especialmente los libros electrónicos. Los bibliotecarios tienen en los títulos de referencia. La imposibilidad de utilizar libros que aprender todas las plataformas en uso por una electrónicos para el préstamo interbibliotecario. biblioteca.

\section{El acceso, control bibliográfico y del uso del libro electrónico}

Se utiliza una base de conocimientos, un resolvedor de enlaces de hipervínculos y una Los títulos adquiridos o suscritos dentro de paquetes alcanzan herramienta de descubrimiento para que resulte el catálogo a través de un resolvedor de enlaces de fácil elegir la plataforma que contiene el libro hipervínculo. Los metadatos asignados a estos elementos son electrónico necesario, encontrar los flujos de muy pobres, a pesar de una ligera mejoría detectada en los trabajo más eficaces y registrar los datos del libro últimos años. Los títulos individuales son catalogados por los por medio de la catalogación por un solo clic.

bibliotecarios cuando se adquieren.
\end{abstract}

\title{
7. CONCLUSIONES
}

Ambas bibliotecas universitarias suecas, independientemente de su tamaño y otras características que las diferencian enormemente, se enfrentan a los mismos desafíos a la hora de adquirir libros electrónicos descritos en la literatura de referencia internacional. Es muy probable que otros problemas de gestión de libros electrónicos, de su preservación y de su uso, también sean similares en todo el mundo, al tratarse de un sector del mercado muy especializado y globalizado. Por otro lado, el uso del libro electrónico en las instituciones de educación superior en Suecia se está volviendo cada día más popular, la demanda de los mismos está creciendo y los libros electrónicos incorporan ciertas ventajas tanto para las bibliotecas como para sus usuarios, destacando entre ellas el acceso ilimitado a múltiples lectores desde cualquier lugar y en cualquier momento, reducción de la necesidad de disponer de grandes espacios físicos (que pueden dedicarse a otros fines), y el hecho de que los textos sean fácilmente transportables por los usuarios en sus ligeros dispositivos de acceso.

La práctica totalidad de los proveedores mencionados en la revisión de ambas bibliotecas ofrecen libros en inglés. Como es el idioma principal que se utiliza en la comunicación científica y aún en muchas áreas de conocimiento es el idioma más utilizado en los textos de lectura universitarios, las necesidades de los usuarios de las bibliotecas universitarias son fácilmente satisfechas a este respecto. Sin embargo, esta situación no está exenta de problemas. Los proveedores de libros electrónicos establecen las condiciones de su utilización y los bibliotecarios han perdido la mayor parte de su libertad de gestión sobre estos títulos en comparación con los libros impresos. Hay barreras significativas para la catalogación de los libros electrónicos y su comercialización eficiente que están relacionados con la enorme diversidad de plataformas y formatos, ya que cada proveedor utiliza su propia plataforma. Las herramientas de descubrimiento de información resultan de uso obligado para que los usuarios sean capaces de acceder al contenido 
de las colecciones de libros electrónicos. La biblioteca tiene que formar a un gran número de bibliotecarios como expertos en plataformas de libros electrónicos de forma separada, ya que cada proveedor tiene su propia plataforma y suelen utilizar formatos propietarios. Los bibliotecarios tienen que organizar talleres de formación y encuentros de trabajo para los usuarios de libros electrónicos por la misma razón. Además, como la existencia de múltiples plataformas puede ser vista por los usuarios como un producto de la comercialización de la imagen de marca de los editores, la promoción de los libros electrónicos y las sesiones de formación pueden perder su eficacia.

Los libros en lengua sueca para las bibliotecas universitarias no están disponibles todavía en formatos de libros electrónicos, a excepción de algunos títulos que pueden ser adquiridos a través de Elib o Adlibris. Los encuestados también mencionaron un proveedor internacional que ofertaba algunos títulos suecos. El problema es más agudo en las disciplinas en las que los profesores recomiendan preferentemente libros de texto suecos (por ejemplo, en Enfermería). Los editores suecos no están dispuestos a ofrecer sus versiones digitales porque temen que los préstamos interbibliotecarios de estos libros de texto puedan afectar sus ingresos. Los bibliotecarios sienten una clara necesidad de tener más libros de texto en formato electrónico y en idioma sueco en algunas disciplinas.

También parece que, en general, los grupos de actores involucrados esperan que los libros electrónicos funcionen como los libros impresos y expresan su insatisfacción entre sí en estos términos. Los editores tratan de suprimir el potencial radical de libro electrónico (Winston, 1998), mediante la introducción de sistemas de gestión de derechos digitales y otros sistemas restrictivos sobre ellos, aniquilando las ventajas básicas de libro electrónico o, al menos, poniéndoles un precio significativamente más alto que a un libro impreso. Para este grupo, un libro electrónico representa una amenaza para el modelo de ingresos tradicional a través de la venta de copias físicas del mismo título a los usuarios. Un libro electrónico, en principio, suprime la necesidad de comprar uno o varios ejemplares de un mismo título y puede ser visto como una fuente única para múltiples réplicas digitales que pueden ser producidas y distribuidas sin mucho esfuerzo o coste.

Por su parte, los bibliotecarios esperan tener posibilidades de gestionar los libros electrónicos tal como solían hacer con los libros físicos: la selección de títulos particulares, el fomento de las colecciones digitales y su crecimiento con el paso del tiempo reflejando los cambios en las demandas de sus comunidades de usuarios. La pérdida de control sobre estas funciones esenciales les hace sentir impotentes e incapaces de llevar a cabo una parte esencial de la misión de la biblioteca. Esta impotencia impide a las bibliotecas la exploración de nuevas posibilidades y averiguar el potencial aún sin explotar de los libros electrónicos académicos. Parece que un libro electrónico como una innovación no encaja orgánicamente en las estructuras tradicionales del mercado, pero tiene que ser forzado a ello. Ninguno de los participantes en el estudio ha mencionado como problema la alta tasa de IVA que se aplica sobre los libros electrónicos (en comparación con los impresos). Esto no parece preocupante para las bibliotecas universitarias suecas, que son organismos estatales y poseen una reducción de la tasa aplicada, sin embargo, debería ser interesante profundizar en esta cuestión, porque con el IVA más alto, los libros electrónicos parecen más atractivos para las administraciones (que tienen tasas reducidas al considerarse un bien cultural y de conocimiento) que para los ciudadanos en general a quienes les llega como un producto comercial, gravado similarmente como cualquier artículo de consumo masivo, despojándolos de otros privilegios de los que gozan los autores que sí existen en las versiones impresas. Esta cuestión tiene un efecto perjudicial sobre el potencial radical de los libros electrónicos y dificulta la implantación de los libros electrónicos en la sociedad.

\section{NOTAS}

\footnotetext{
${ }^{1}$ Ver los sitios web http://www.knowledgeunlatched.org o http://www.oapen.or

${ }^{2}$ Winston enuncia un principio pseudosociológico considerando que la sorpresa que causa un avance en el campo de las telecomunicaciones es equivalente al esfuerzo que hará falta para cambiar los hábitos de uso de inventos anteriores, eso es a lo que él llama "suprimir el potencial radical". ${ }^{3}$ Más información en http://jwikert.typepad.com/

${ }^{4}$ SFX es básicamente un resolvedor de enlaces de hipertexto que proporciona al usuario de una biblioteca, de forma ágil y rápida, todas las opciones de acceso que esta biblioteca dispone para un recurso informativo determinado.
}

\section{Agradecimientos}

Los autores agradecen el apoyo de la agencia Swedish Research Counsil, entidad que financia el proyecto "The impact of the e-book on 'small language' culture: media technology and the digital society" $\mathrm{y}$ de las dos bibliotecas universitarias y de su personal que generosamente han apoyado los estudios de caso, por su tiempo y entusiasmo. 


\section{BIBLIOGRAFÍA}

AHMAN, P. y BROGAN, M. Scholarly use of e-books in a virtual academic environment: a case study. Australian Academic y Research Libraries, 2012, vol. 43, n 3, p. 189-213.

ARMSTRONG, C. Books in a virtual world: the evolution of the e-book and its lexicon. Journal of Librarianship and Information Science, 2008, vol. 40, nº 3, p. 193-206.

ARMSTRONG, C. y LONSDALE, R. E-books basics. London: UKeiG: UK einformation Group, 2014 [en línea]. Disponible en: <http://www.webcitation.org/6PIPYQjg6> [Consulta: 20 de marzo de 2015].

ARMSTRONG, C. y LONSDALE, R. Challenges in managing e-books collections in UK academic libraries. Library Collections, Acquisitions and Technical Services, 2005, vol. 29, $\mathrm{n}^{\circ}$ 1, p. 33-50.

ASHCROFT, L. Ebooks in libraries: an overview of the current situation. Library Management, 2011, vol. 32, ${ }^{\circ}$ 6/7, p. 398-407.

BEISLER, A. y KURT, L. E-book workflow from inquiry to access: facing challenges to implementing e-book access at the University of Nevada, Reno. Collaborative Librarianship, 2012, vol. 4, n 3, p. 96-116.

BENNETT, L. y LANDONI, M. E-books in academic libraries. The Electronic Library, 2005, vol. 23, nº 1, p. 9-16.

BERGSTRÖM, A.; HÖGLUND, L.; MACEVICIUTE, E.; NILSON, K. y WILSON, T.D. The case of the e-book in a 'small language' culture: media technology and the digital society. Knygotyra, vol. 62, 2014 [en línea]. Disponible en: <http://www.zurnalai.vu.lt/files/journals/51/articles/3608/public/73-93.pdf> [Consulta: 17 de marzo de 2015].

BERNHARDSSON, K.; ERIKSSON, J.; HENNING, K.; LINDELÖW, C.H.; LAWRENCE, D.; NEIDENMARK, T. y SVENSSON, A. A National Consortium for Open Academic Books in Sweden. An investigation funded by National Library of Sweden, Swedish Research Council and Riksbankens Jubileumsfond. Final report. Stockholm: National Library of Sweden, 2013 [en línea]. DOI: http://dx.doi.org/10.3384/liu:diva-94273.

BLUMMER, B. y KENTON, J. Best practices for integrating e-books in academic libraries: a literature review from 2005 to present. Collection Management, 2012, vol. 37, $\mathrm{n}^{\circ}$ 2, p. 65-97.

BUCKNELL, T. Buying by the bucketful: a comparative study of e-book acquisition strategies. Insight: the UKSG journal, 2012, vol. 25, $\mathrm{n}^{\mathrm{o}} 1$, p. 51-60.

BURNETTE, E.S. Budgeting and acquisitions. En: COLLINS, M.D.D. y CARR. P.L. (Eds.), Managing the transition from print to electronic journals and resources. Oxford: Routledge, 2008, p. 3-28.

BYSTRÖM, K. Everything that's wrong with e-book statistics: a comparison of e-book packages. En: BERNHARD, B.R., HINDS, L.H. y STRAUCH K.P. (Eds), Accentuate the positive: Charleston Conference Proceedings 2012. West Lafayette, Indiana: Purdue University Press, 2012, p. 216-220 [en línea]. Diponible en: $<\mathrm{http} / / /$ docs.lib.purdue.edu/cgi/viewcontent.cgi? article=1362ycontext=charleston $>$ [Consulta: 20 de marzo de 2015].

CASSIDY, E.D.; MARTINEZ, M. y SHEN, L. Not in love or not in the know? Graduate student and faculty use (and non-use) of e-books. The Journal of Academic Librarianship, 2012, vol. 38, nº 6, p. 326-332.

DEVENNEY, A. y CHOWCAT, I. Research underpinning the SCONUL and JISC e-books co-design project. 2013 [en línea]. Disponible en: <http://bit.ly/lnYx1rX> [Consulta: 16 de marzo de 2015].

DEWAN, P. Are books becoming extinct in academic libraries? New Library World, 2012, vol. 113, n 1/2, p. $27-37$.

FISCHER, K.S.; WRIGHT, M.; CLATANOFF, K.; BARTON, H. y SHREEVES, E. Give 'em what they want: a oneyear study of unmediated patron-driven acquisition of e-books. College y Research Libraries, 2012, vol. 73, $\mathrm{n}^{\circ} 5$, p. 469-492.

FREDRIKSEN, L.; CUMMINGS, J.; CUMMINGS, L. y CARROLL, D. Ebooks and interlibrary loan? Licenced to fill. Journal of Interlibrary Loan, Document Delivery y Electronic Reserve, 2011, vol. 21, nº 3, p. 117-131.

GUSTAF, H. E-böcker på högskole-och universitetsbibliotek. En studie kring det digitala förvärvet: kandidatsuppsats i biblioteks- och informationsvetenskap. [E-books in college and university libraries: a study of digital acquisition]. [Unpublished Bachelor's dissertation]. Borås: University, 2010.

HODGES, D.; PRESTON, C. y HAMILTON, M. Resolving the challenge of e-books. Collection Management, 2010, vol. $35, n^{\circ} 3$, p. 196-200.

KONRAD, K. Old habits in a new world? E-book management techniques at an academic library. [Unpublished Master dissertation]. Borås: University, 2013.

KULCZYCKI, E. eBook jako monografia naukowa - czyli kiedy moge dostać punkty? [the eBook as a scientific monograph - that is, when do I get points?]. 2012 [en línea]. Disponible en: $<$ http://www.webcitation.org/6PSoU6ijE > [Consulta: 20 de marzo de 2015].

KUNGLIGA BIBLIOTEKET. När kommer boomen? En kartläggningav e-boken i Sverige ur ettbiblioteksperspektiv. [When will the boom happen? A survey of the e-book in Sweden from a library perspective]. Stockholm: SvenskBiblioteksföreningen, 2011.

KUNGLIGA BIBLIOTEKET. Forskningsbibliotek 2002-2012 tabell NHS1-NHS8. [Research libraries 2002-2012, tables NHS1-NHS8]. Stockholm: Kungliga Biblioteken, 2012. 
KUZMINIENE, R. E-books in Irish universities' libraries: changes and challenges in collection development and acquisitions. [Unpublished Master's dissertation]. Borås: University, 2014.

LIPPINCOTT, S.K.; BROOKS, S.; HARVEY, A.; RUTTENBERG, J.; SWINDLER, L. y VICKERY, J. Librarian, publisher, and vendor perspectives on consortial e-book purchasing: the experience of the TRLN beyond print summit. Serials Review, 2012, vol. 38, nº 1, p. 3-11.

MACEVICIUTE, E. y BORG, M. The current situation of e-books in academic and public libraries in Sweden. Libellarium, 2013, vol. 6, n 1-2, p. 13-28.

MARTIN, K. y QUAN-HAASE, A. Are e-books replacing print books? tradition, serendipity, and opportunity in the adoption and use of e-books for historical research and teaching. Journal of the American Society for Information Science and Technology, 2013, vol. 64, n 5, p. 1016-1028.

MEDEIROS, N. Shaping a collection one electronic book at a time: patron-driven acquisition in academic libraries. OCLC Systems y Services: International digital library perspectives, 2011, vol. 27, $\mathrm{n}^{\circ}$ 3, p. 160-162.

MEEHAN, D. Strictly online? E-books in the academic world. Panlibus Magazine, 2010, vol. 17, nº 8-9.

MINČIĆ-OBRADOVIĆ K. E-books in academic libraries. Oxford: Chandos, 2011.

MORRIS, C. y SIBERT, L. Acquiring e-books. En: POLANKA, S. (Ed.), No shelf required: E-books in libraries, Chicago: American Library Association Editions, 2010, p. 95-124.

MULVIHILL, A. Librarians face e-book acquisition obstacles. Information Today, 2011, vol. 28, no 5, p. 13.

MÜLLER, H. Legal aspects of e-books and interlibrary loan. Interlending y Document Supply, 2012, vol. 40, nº 3, p. 150-155.

NELSON, M.R. E-books in higher education: nearing the end of the era of hype? EDUCAUSE Review, 2008, vol. 43, $\mathrm{n}^{\circ}$ 2, p. 40-56 [en línea]. Disponible en: <http://www.educause.edu/ero/article/e-books-higher-education-nearingend-era-hype $>$ [Consulta: 18 de marzo de 2015].

NELSON, M.R. y HAINS, E. E-books in higher education: are we there yet? ECAR Research Bulletin, 2010, vol. 2, p. 1-13 [en línea]. Disponible en: < https://net.educause.edu/ir/library/pdf/ERB1002.pdf $>$ [Consulta: 18 de marzo de 2015].

RADNOR, M.C. y SHRAUGER, K.J. E-book resource sharing models: borrow, buy, or rent. Journal of Interlibrary Loan, Document Delivery, 2012, vol. 22, n 3-4, p. 155-161.

SCHELL, L. The academic library e-book. En: POLANKA, S. (Ed.). No shelf required: e-books in libraries. Chicago: American Library Association Editions, 2010, p. 75-94.

SHEPHERD, J. y ARTEAGA R. Social work students and e-books: a survey of use and perception. Behavioural and Social Sciences Librarian, 2014, vol. 33, $\mathrm{n}^{\circ} 1$, p. 15-28.

SÖDERBACK, A. Infrastructure first! E-books and academic libraries in Sweden. Serials, 2011, vol. 24, n 1 , p. 3842.

SRIVASTAVA, R. y SHARMA, B. Digital rights management. En: Publicon 2012. Digital Publishing, September 34, 2012. FICCI, Federation House. Selected Papers, 2012, p. 46-57. New Delhi: FICCI, Zubaan Books [en línea]. Disponible en: $<$ http://bit.ly/1jJPuo2> [Consulta: 18 de marzo de 2015].

STAMISON, C.M. Developing a sound e-book strategy. Information Outlook, 2011, vol. 15, no 5, p. 10-12.

SVEDJEDAL, J. Biblioteken och bokmarknaden - från folkskolan till e-böcker [Libraries and the book market - from elementary school to e-books]. Stockholm: Svenska Förläggareföreningen, 2012.

SVENSSON, A. y ERIKSSON J. Monographs and open access. Sciencominfo (Nordic-Baltic Forum for Scientific Communication), 2013, vol. $9, \quad \mathrm{n}^{\mathrm{o}} 1$ [en línea]. Disponible en: $<$ http://journals.lub.lu.se/ojs/index.php/sciecominfo/article/view/6126> [Consulta: 18 de marzo de 2015].

SWEDEN. MINISTRY OF CULTURE. Läsandets kultur. Slutbetänkande av litteraturutredningen [Reading culture: final report of the literature survey]. Stockholm: Ministry of Culture, 2012. Disponible en: $<$ http://www.regeringen.se/content/1/c6/20/02/57/65903c80.pdf $>$ [Consulta: 18 de marzo de 2015].

SWEDEN. PARLIAMENT. En bok är en bok är en bok? - en fördjupningsstudie av e-böckerna i dag [A book is a book is a book? In-depth study of e-books today]. Stockholm: Riksdagstrykeriet, 2013. Disponible en: $<$ http://data.riksdagen.se/fil/F358F77F-BAD8-4B71-8F86-B9D0140A80F0> [Consulta: 21 de marzo de 2015].

TEDD, L.A. E-books in academic libraries: an international overview. New Review of Academic Librarianship, 2005 , vol. $11, \mathrm{n}^{\mathrm{o}} 1, \mathrm{p} .57-79$.

TEDD, L.A. y CARIN, W. Selection and acquisition of e-books in Irish institutes of technology libraries. Aslib Proceedings, 2012, vol. 64, $\mathrm{n}^{\circ}$ 3, p. 274-288.

THOMPSON, S. y SHARP, S. E-books in academic libraries: lessons learned and new challenges. Serials, 2009 , vol. 22, $\mathrm{n}^{\circ} 2$, p. 136-140.

TYLER, D.; FALCI, C.; MELVIN, J.; EPP, M. y KREPS, A. Patron-driven acquisition and circulation at an academic library: interaction effects and circulation performance of print books acquired via librarians' orders, approval plans, and patrons' interlibrary loan requests. Collection management, 2013, vol. 38, nº 1, p. 3-32.

VASILEIOU, M. y ROWLEY, J. Marketing and promotion of e-books in academic libraries. Journal of Documentation, 2011, vol. 67, $\mathrm{n}^{\circ}$ 4, p. 624-643. 
VASILEIOU, M.; HARTLEY, R. y ROWLEY, J. Choosing e-books: a perspective from academic libraries. Online Information Review, 2012a, vol. 36, n 1, p. 21-39.

VASILEIOU, M.; HARTLEY, R. y ROWLEY, J. Perspectives on the future of e-books in libraries in universities. Journal of Librarianship and Information Science, 2012b, vol. 44, $\mathrm{n}^{\circ}$ 4, p. 217-226.

VASILEIOU, M.; HARTLEY, R. y ROWLEY, J. The e-book management framework: The management of e-books in academic libraries and its challenges. Library and Information Science Research, 2012c, vol. 34, $\mathrm{n}^{\mathrm{o}} 4$, p. 282291.

VASSILIOU, M. y ROWLEY, J. Progressing the definition of "e-book". Library Hi Tech, 2008, vol. 26, n 3, p. 355368.

VELAGIĆ, Z. The discourse on printed and electronic books: analogies, oppositions, and perspectives. Information Research, 2014, vol. 19, no 2. Disponible en: < http://informationr.net/ir/19-2/paper619.html> [Consulta: 21 de marzo de 2015].

WALTERS, W. H. Patron-driven acquisition and the educational mission of the academic library. Library Resources $y$ Technical Services, 2012, vol. 56, n 3, p. 199-213.

WALTERS, W.H. E-books in academic libraries: challenges for acquisition and collection management. Portal: Libraries and the Academy, 2013, vol. 13, $\mathrm{n}^{\circ}$ 2, p. 187-211. Disponible en: <http://bit.ly/1qt7GYe> [Consulta: 21 de marzo de 2015].

WALTERS, W. H. E-books in academic libraries: challenges for sharing and use. Journal of Librarianship and Information Science, 2014, vol. 46, nº 2, p. 85-95. DOI: http://dx.doi.org/10.1177/0961000612470279.

WIKERT, J. It's time for a unified e-book format and the end of DRM: proprietary e-book formats and rights restrictions are holding customers back [en línea]. 2012. Disponible en: <http://toc.oreilly.com/2012/02/unifiedebook-format- end-drm.html> [Consulta: 18 de marzo de 2015].

WINSTON, B. Media technology and society: a history from the telegraph to the Internet. London: Routledge, 1998. 